\title{
Evaluation of clinical, pathological and genetic indicators in chickens with thiram-induced tibial dyschondroplasia
}

\author{
Hui Zhang ${ }^{1, *}$, Khalid Mehmood ${ }^{1,2, *}$, Kun Li $^{1}$, Wangyuan Yao ${ }^{1}$, Mujeeb Ur Rehman ${ }^{1}$, \\ Fazul Nabi ${ }^{1}$, Houqiang Luo ${ }^{1}$, Muhammad Kashif Iqbal ${ }^{1}$, Muhammad Shahzad ${ }^{1,2}$ and \\ Jiakui Li ${ }^{1,3}$ \\ ${ }^{1}$ College of Veterinary Medicine, Huazhong Agricultural University, Wuhan 430070, PR China \\ ${ }^{2}$ University College of Veterinary and Animal Sciences, Islamia University of Bahawalpur, Bahawalpur 63100, Pakistan \\ ${ }^{3}$ College of Animals Husbandry and Veterinary Medicine, Tibet Agricultural and Animal Husbandry University, Linzhi, Tibet \\ 860000, PR China \\ *These authors contributed equally to the work \\ Correspondence to: Jiakui Li, email: lijk210@sina.com \\ Keywords: tibial dyschondroplasia; indicators; clinical diagnosis; growth plate; chickens \\ Received: June 03, $2017 \quad$ Accepted: December 23, $2017 \quad$ Published: December 29, 2017 \\ Copyright: Zhang et al. This is an open-access article distributed under the terms of the Creative Commons Attribution License 3.0 \\ (CC BY 3.0), which permits unrestricted use, distribution, and reproduction in any medium, provided the original author and source \\ are credited.
}

\section{ABSTRACT}

Tibial dyschondroplasia (TD) is one of the most common leg problems disease in fast-growing birds in the poultry industry. However, no systematic study on the changes in performance indicators of TD chickens during different periods of development has been performed. A total 200 broiler chicks were randomly divided into two equal groups: the normal group and the TD group (induce with $50 \mathrm{mg} / \mathrm{kg}$ thiram). After the induction of TD, the mortality rate, performance indicators, tibia bone indicators, physiological index changes in the serum, and gene and protein expression levels in the growth plate were examined. The results showed that the mortality and feed conversion ratio were higher in the TD group than in the normal group. The length, width and weight of the tibia were of low quality in TD chickens, while the width of the tibial growth plate was significantly enlarged $(P<0.05)$ in the TD group, especially on day 14 post-hatch. The levels of superoxide dismutase (SOD), total antioxidant capacity (T-AOC), glutathione peroxidase (GSH-PX), and alkaline phosphatase (ALP) were significantly decreased, while the levels of malondialdehyde (MDA), were significantly increased in TD chickens compared to normal chickens. Gene expression and western blotting analysis revealed up-regulation of Hsp90 and VEGF expression and down-regulation of Wnt4 expression in TD chickens compared to those in normal chickens. This systemic study compare clinical, pathological and genetic indicators in thiram-induced tibial dyschondroplasia and normal chickens during different age periods, which reveals new targets for the clinical diagnosis and prevention of TD in broiler chickens.

\section{INTRODUCTION}

Tibial dyschondroplasia (TD) is an important tibiotarsal bone disease in fast-growing birds that disturbs the proximal tibial growth plate [1]. TD is characterized by an avascular and non-mineralized growth plate, tibia bone deformation, gait disorders, difficulty in standing, reduced growth and lameness $[2,3]$. Previous research has indicated that almost $30 \%$ of bone disease in chickens and turkeys is due to TD and that the disease leads to greater than $10 \%$ morbidity in China, creating serious economic losses in the poultry industry [4-6]. Normally, the clinical and subclinical rate of TD is $30 \%$ in chickens, which decreases disease resistance, production performance, and carcass quality and induces breast cysts, osteomyelitis, and animal welfare issues [7-9]. 
Studies have shown that angiogenesis and vascular development are inhibited in osteoblasts, osteoclasts and mesenchymal stem cells, so calcified cartilage cannot complete the bone sedimentary process, which leads to white cartilage suppository $[10,11]$. In TD, a large amount of the cartilage cell damage is due to the apoptosis process and abnormal protein secretion in cartilage cells, causing a decrease in the cartilage extracellular matrix degradation rate, which limits the space for bone deposition $[12,13]$. Currently, research focuses on increasing the growth rate and feed conversion ratio (FCR) of broilers; consequently, poultry bone disease incidence is also increasing in the broiler industry $[5,14,15]$. It is reported that the chicken muscle tissue and bone growth and development destroy the original balance and are also a reason for the leg deformities in chicken [16]. Normal growth plate development requires cartilage vascularization and mineralization followed by osteogenesis, while in tibial dyschondroplasia, the differentiation of chondrocytes appears to be abnormal $[7,11]$.

The heat-shock protein 90 (Hsp90), a molecular chaperone protein that mediates protein folding, stability and maturation, is obligatory for cartilage development and differentiation. In our previous studies, we found that Hsp90 is highly expressed in TD lesions $[1,17]$. Wnt4 is a secreted protein, and some research has shown that Wnt4 from osteoblasts significantly protects against bone loss by inhibiting osteoclast formation and bone resorption $[18,19]$. Vascular endothelial growth factor (VEGF) is the critical pro-angiogenic factor for vascular formation [20]. Analysis of the cellular morphology of different regions of the growth plate revealed that cells surround the large blood vessels in the hypertrophic zone. However, thiram caused morphological and histological changes characterized by a significantly thickened avascular cartilage plug, dry chondrocytes, and empty cartilage lacunae. Moreover, the particular arrangement and shape of the cells were lost due to the lack of blood vessels in the TD-affected growth plate [21]. Endochondral ossification is a completely connected and controlled mechanism that is regulated via the proliferation and differentiation of epiphyseal growth plate chondrocytes [22]. Birds in the TD group developed a lesion filled with cartilaginous material, but when the thiram supplement was stopped, the lesion started decreasing, and the growth plate underwent a self-recovery process and was replaced by bone $[8,9]$. Therefore, this study was conducted to understand the mechanism of self-healing in thiraminduced growth plates.

Serum levels of SOD, MDA, GSH-Px, and T-AOC and the levels of AST, AKP and ALP reflect the damage in the livers in chickens $[1,23]$. Although, each index changes in chickens when TD occurs, few studies have shown the change in these indicators during TD. The aim of our study was to evaluate clinical, pathological and genetic indicators in thiram-induced tibial dyschondroplasia and normal chickens, which will identify therapeutic targets and provide a reference for the early diagnosis of TD.

\section{RESULTS}

\section{Chicken mortality rate assay}

The mortality rates in the normal and TD group were $3 \%(3 / 100)$ and $11 \%(11 / 100)$, respectively, from day 7 to day 18 . The rate of mortality on day 7 and 10 was greatly increased in the TD group compared with that in the normal group, and the difference was statistically significant $(P<0.05)$ in both groups. The maximum mortality appeared at day 10 in the TD group and then begin to decrease. Additionally, the results showed that TD chickens have a nearly 4.0 fold higher mortality risk than the control group (Figure 1).
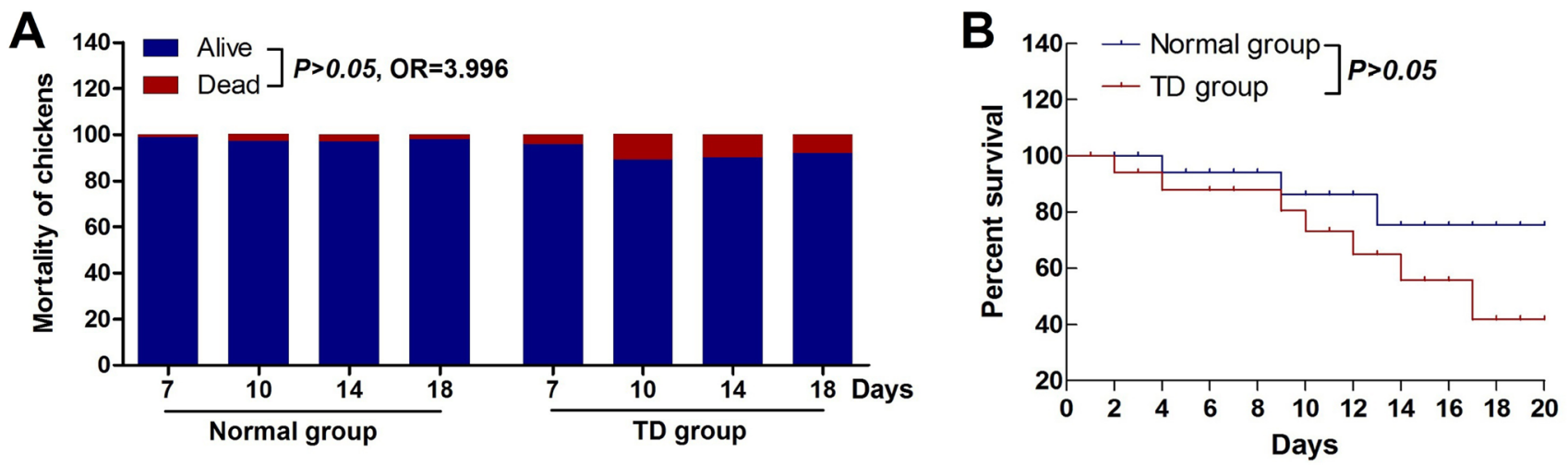

Figure 1: The mortality and percent survival in the normal and TD group. (A) Difference in mortality between normal and TD chickens $(n=100)$. Chi-square analysis was performed on the number of chickens who died during the experiment. The mortality curve was produced with Prism software (GraphPad, San Diego, CA, USA). Five hundred and forty-seven comparisons were evaluated with the log rank (Mantel-Cox) test for the mortality curve (each curve represents 100 chicks). (B) Percent survival between chickens in the normal and TD group. Chi-square analysis was performed on the number of chickens who remained alive during the experiment. (Odds Ratio $=3.996 ; 95 \%$ Confidence Interval $=0.92 \sim 16.66)$. 


\section{Performance parameter analysis of chickens}

The overall performance of the chicken growth indicators are given in Figures 2 and 3. The results indicated that lameness was increased significantly throughout the experiment in the TD group of chickens compared with the normal group. However, a higher degree of lameness was recorded on day 7 and 10, and after that, it started to decrease, while the normal group of chickens remained healthy throughout the experimental period without showing any signs of lameness (Figure 2).

Analysis of chicken performance parameters showed that there was a positive correlation among the weight of chickens, average daily feed intake and average daily weight gain, while a negative correlation with the feed conversion ratio over the entire experimental period in the normal and TD group. The weight of chickens, average daily feed intake and average daily weight gain were less in the TD group than in the normal group, and there was a significant difference $(P<0.05)$ between the two groups (Figure 3A, 3B and 3C). However, the feed conversion ratio was significantly higher than the reference value in TD chickens compared with normal chickens $(P<0.05)$ during the experimental period (Figure 3D), which indicated poor FCR.

\section{Performance parameter analysis of the tibia bone, growth plate and TD score}

The overall performance of tibia bone indicators is given in Figures 4 and 5. Morphological examination of the proximal tibial growth plate (GP) in the normal and TD group indicated that the growth plate of TD chickens was remarkably increased compared to normal chickens during the experimental period from day 7 to day 14 , and then, it started to decrease (Figure 4A). However, the normal group did not show any change in the growth plate during the study period. The TD score indicated the severity of disease and lameness in TD and normal chickens during the experimental period (Figure 4B). The average and relative scores were higher in the TD group than in the normal group during the experimental period.

Overall performance parameter analysis of the tibia bones showed that there was a positive correlation among the length of the tibia, width of the tibia bone and weight of the tibia, while a negative correlation was observed in the width of the tibial growth plate during the entire experimental period in the normal and TD group. The length, width and weight of the tibia bones were significantly decreased $(P<0.05)$ in the TD group compared with those in the normal group (Figure 5A-5C). However, the width of the GP was significantly increased $(P<0.05)$ during the experimental period in the TD group than in the normal group (Figure 5D). In the normal group, the size of the GP was between 0.1$0.2 \mathrm{~cm}$ throughout the experimental period. Additionally, our study also found that the ratio of tibia length to chicken weight (TB. length/C. weight) and the ratio of tibia weight to chicken weight (TB. weight/C. weight) were clearly lower in the normal group than in the TD group (Figure 5F-5G).

\section{Histology of the tibial growth plate with hematoxylin \& eosin staining}

Histology of the chicken tibial growth plates showed that the normal group had well-conserved columns surrounded by massive blood vessels in the proliferative and hypertrophic zones of the GP (Figure 6). However, marked alterations in the growth plate of the TD chickens were noticeable, with shrinking cells and

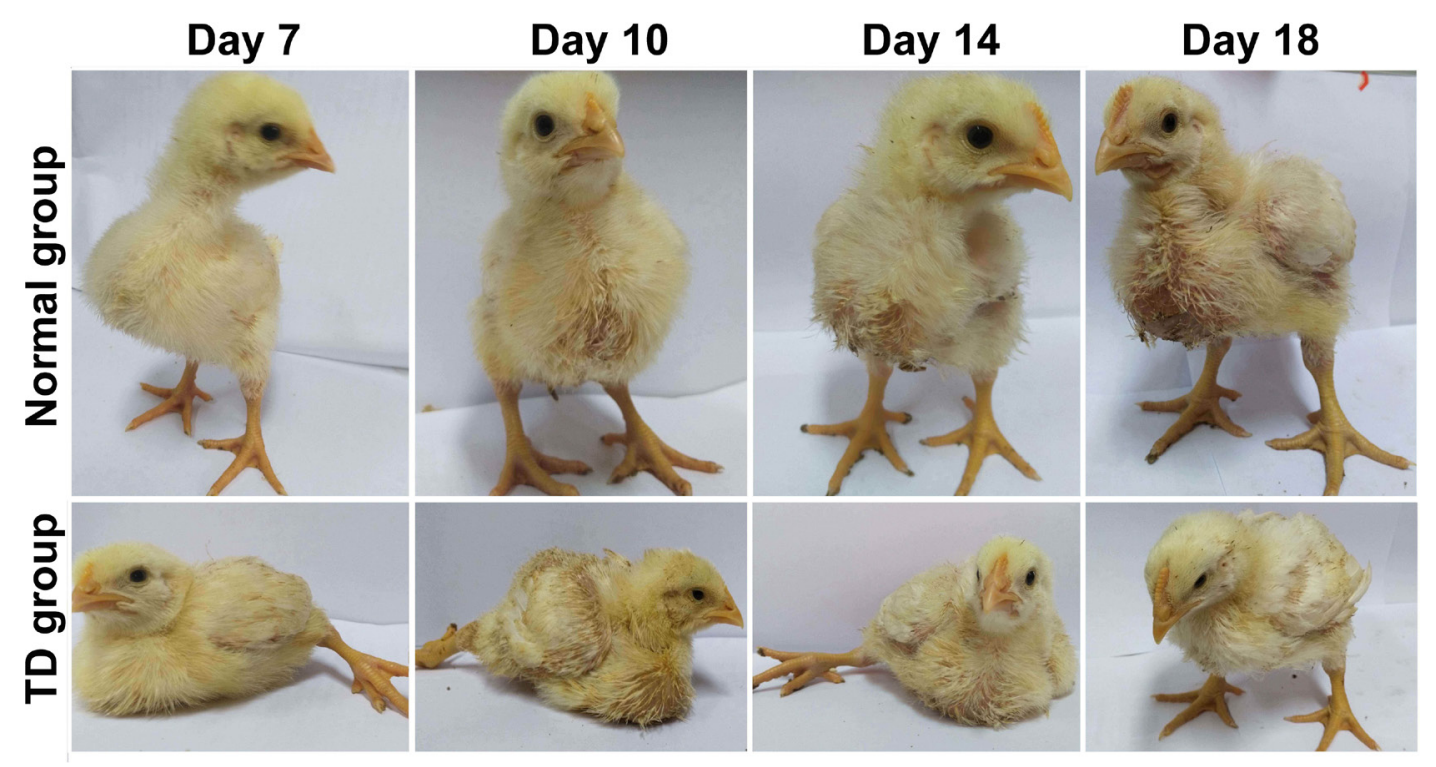

Figure 2: Normal and TD Chickens were photographed throughout the experiment. Lameness was significant in the TD group during the experimental period, but no lameness was observed in the normal group. 
irregular chondrocyte columns. Therefore, we next asked whether the development of the tibia is correlated with vascular distribution in the tibial growth plate. Histological assessments showed that vascular distribution decreased during the experimental period in the TD group compared with that in the normal group. Simultaneously, the statistical data showed that the number of blood vessels was decreased in the TD group, as shown in the histological pictures in Figure 6. In addition, the number and area of blood vessels are strongly correlated with the length, width and weight of the tibia bone. Hence, these observations demonstrated that tibial vascular distribution was decreased in the TD group compared with that in the normal group.

\section{Biochemical criterion analysis of serum}

Our experiment showed a significant decrease $(P<0.05)$ in ALP activity along with an increase in ALT and AST content in the serum of the TD group during the entire experimental period. However, the levels of ALT and AST were significantly higher, while ALP activity was significantly lower $(P<0.05)$ in the TD group than in the normal group. Altogether, the differences between the TD group and the normal group (Table 1) were significant $(P<0.05)$ throughout the experimental period.

\section{Liver antioxidant levels}

In our study, the activity levels of SOD, GSH-Px and T-AOC were significantly decreased $(P<0.05)$, while MDA content was increased in the livers of TD chickens compared to those in normal chickens. The activity levels of SOD and GSH-Px had an increasing trend in both groups from day 7 to day 18 . However, the T-AOC activity decreased in TD group, while it increased in the normal group from day 7 to day 18. Low antioxidant levels were found in the TD group compared with those of the normal group (Table 2).
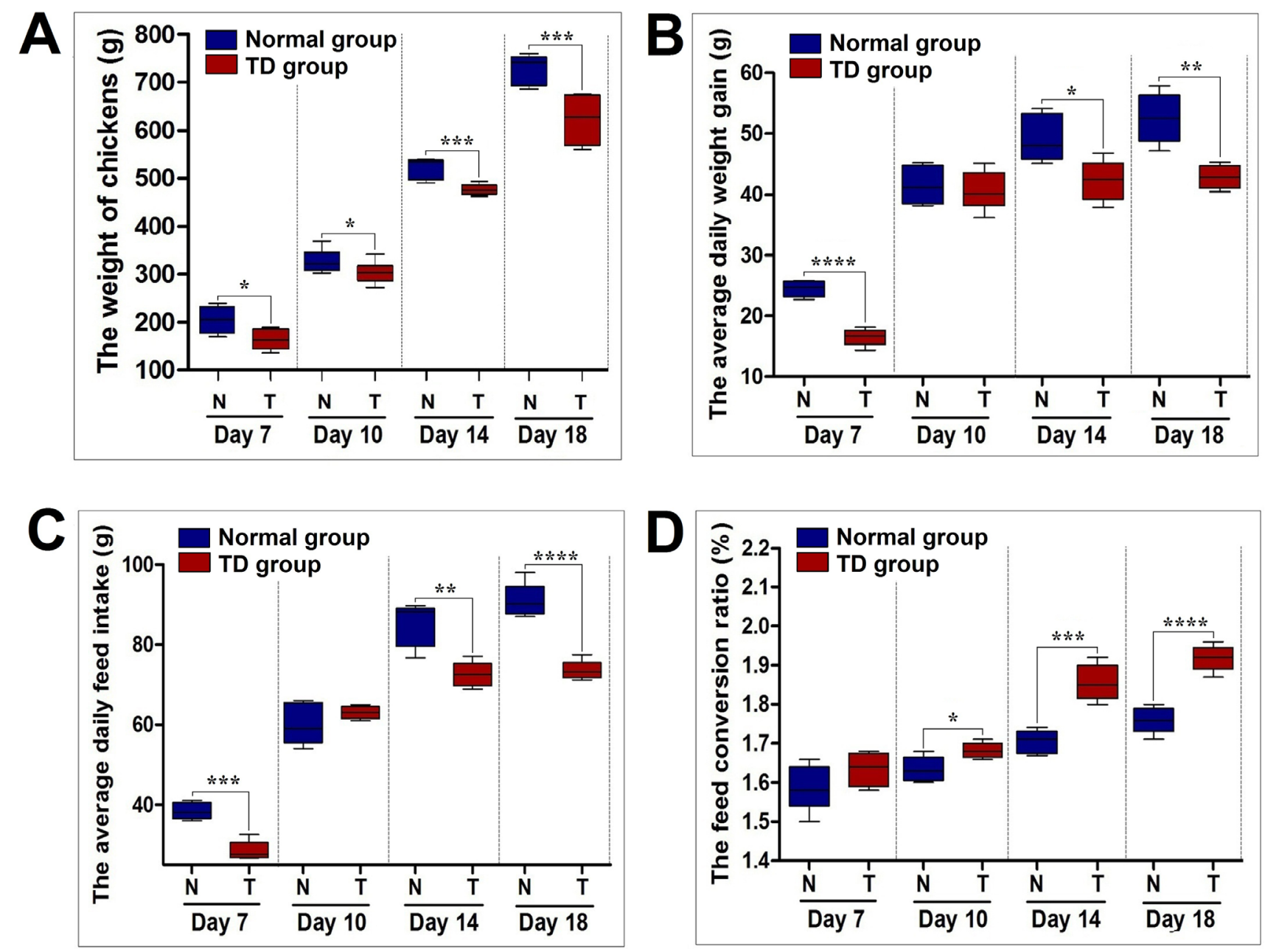

Figure 3: Overall performance parameter analysis of normal (N) and TD (T) chickens. (A-D) The correlation of the weight of the chickens, average daily feed intake, average daily weight gain and feed conversion ratio was analyzed with Pearson's test in the normal and TD group during the experiment from day 7 to day 18. The reference (standard) value of FRC is 1.6 in this study figure. ${ }^{*} P<0.05,{ }^{* *} P<0.01,{ }^{* * *} P<0.001,{ }^{* * * *} P<0.0001$. 


\section{RT-qPCR analysis of Hsp90, Wnt4 and VEGF genes}

The results showed a significant increase $(P<0.05)$ in the mRNA expression level of Hsp90 from day 7 to day 14 in the tibial growth plate of the TD group compared with that of the normal group (Figure 7A). This finding indicated that Hsp90 plays an important role in stress in TD chickens. The mRNA expression level of the Wnt4 gene significantly decreased $(P<0.05)$ from day 7 to day 14 in the tibial growth plate of the TD group compared with the normal group (Figure 7B). Therefore, the tibia bone became significantly more deformed and resorbed in the TD chickens than in the normal chickens. To explore the transcriptional and translational levels of angiogenesisrelated genes in the tibial GP of TD and normal chickens, we performed RT-qPCR for the VEGF gene using the tissues obtained from the early phase tibia. As shown in Figure $7 \mathrm{C}$, a significant increase $(P<0.05)$ in the mRNA expression level of the VEGF gene was observed
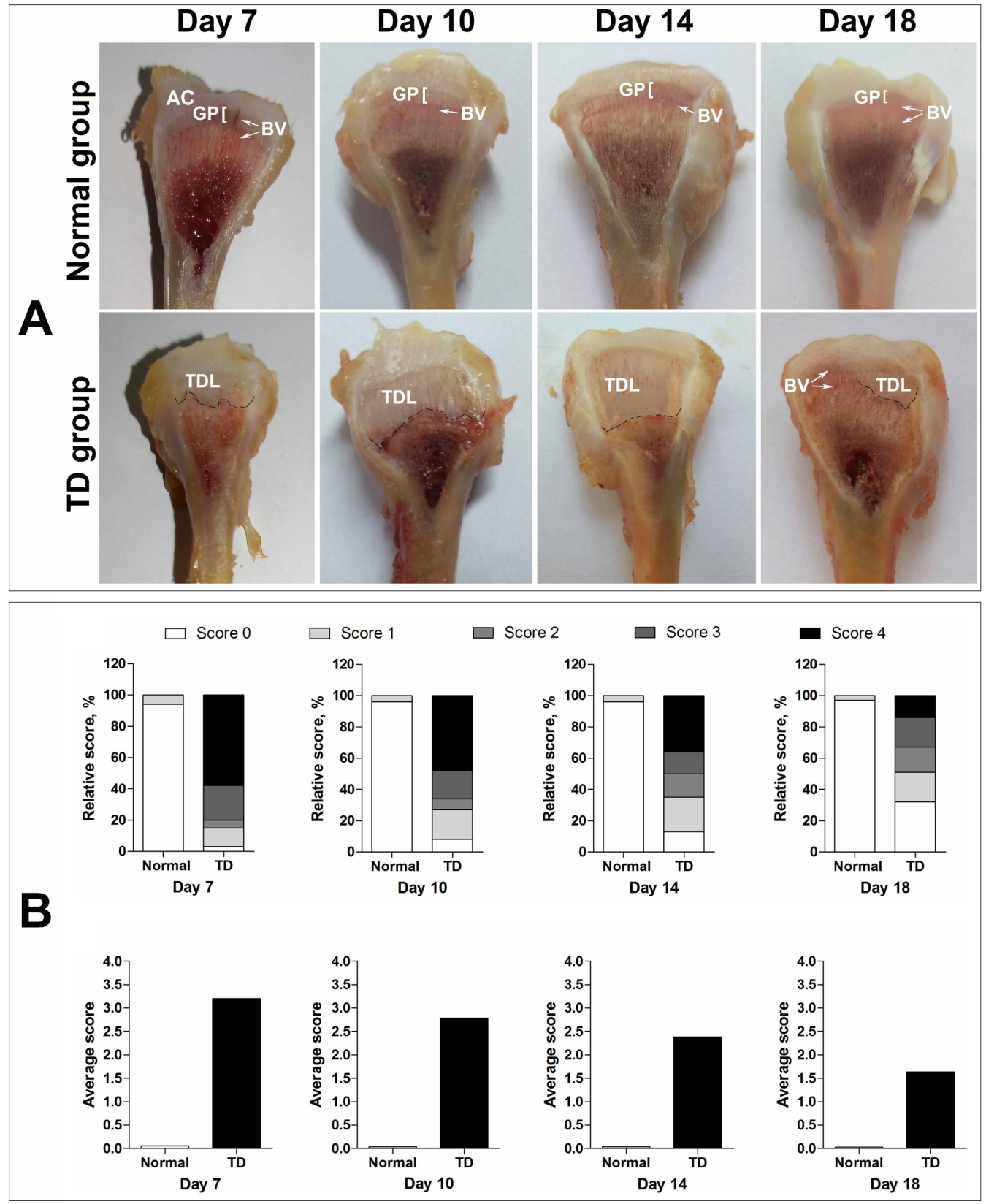

Figure 4: Severity of TD in thiram-fed chickens. (A) The morphological examination of proximal tibial growth plates (GP) in the normal group and the TD group. GPs were extended in the TD group compared to the normal group during the experimental period from day 7 to day 18. (B) The effect of thiram on tibial dyschondroplasia (TD) incidence. All tibial growth plates were dissected to compare the average and the relative scores on day 7, 10,14 and 18 in the TD and normal group. 
in the tibial GP of the TD group compared to that in the normal group from day 7 to day 14 . On day 18 , the mRNA expression level of the VEGF gene in the TD group was nearly equal to that of the normal group. We found positive correlation in gene expression between Hsp90 and VEGF, while negative correlation in WNT4 genes (Figure 7D-7F).

\section{Western blot analysis of Hsp90, Wnt4 and VEGF proteins}

Western blotting further identified that the Hsp90 and VEGF proteins in the tibia were significantly upregulated in the TD group growth plates on days 7, 10 and 14 compared with those in the normal group (Figure 8A and 8C). Statistical analysis of the western blots revealed a significant increase $(P<0.05)$ in the expression level on days 7,10 and 14 , but it was restored to the level of the normal group on day 18. In contrast, there were marked reductions in the Wnt4 expression level in the growth plates of the TD chickens on days 7, 10 and 14 . The statistical analysis showed that the expression level of Wnt4 was significantly decreased $(P<0.05)$ on days 7,10 and 14, but it was restored to normal on day 18 (Figure 8B). Furthermore, western blotting analysis of the VEGF results were also parallel to the significant gene expression results, as shown in Figure 8C.

\section{DISCUSSION}

The current study showed that the mortality rate was significantly higher $(P<0.05)$ in the TD group than in the normal group. The performance parameters of TD chickens indicated a reduction in the average daily feed intake and average daily weight gain along with a poor feed conversion ratio compared with the normal chickens, and these differences were significant $(P<0.05)$ on day 10 between the two groups. The length, width and weight of the tibia bone were significantly decreased in the TD
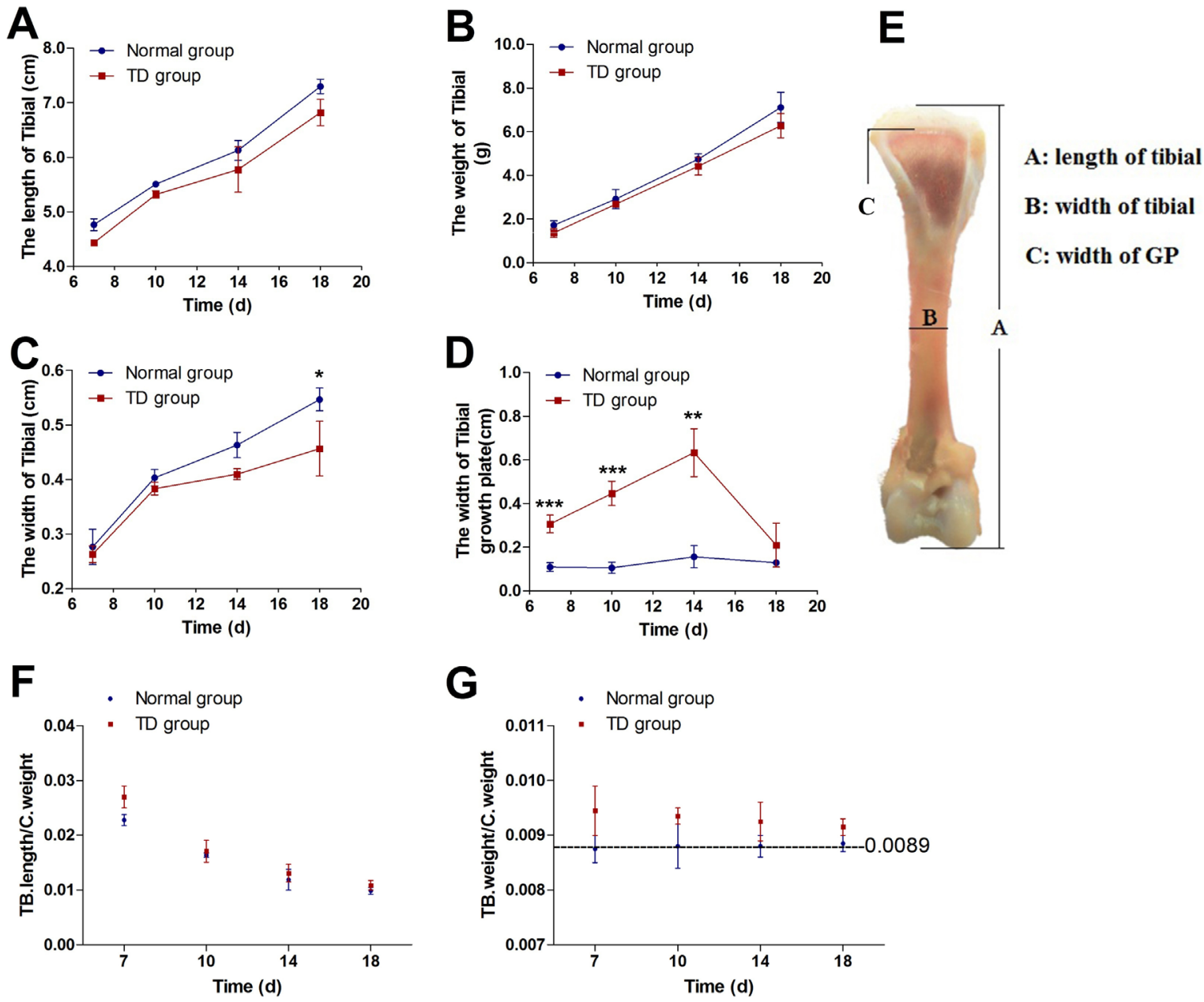

Figure 5: Overall performance analysis of tibia bone indicators was performed in normal and TD chickens. (A-G) Correlation analysis of the length of the tibia, width of the tibia, weight of the tibia, width of the tibial growth plate, the ratio of tibia length per chicken weight (TB. length/C. weight) and the ratio of tibia weight per chicken weight (TB. weight/C. weight) was recorded with Pearson's test in the normal and TD group during the experiment from day 7 to day $18 .{ }^{*} p<0.05,{ }^{* *} p<0.01,{ }^{* * *} p<0.001$, Error bars indicate SD. 
group compared with the normal group, while the width of the tibial growth plate was significantly larger, especially on day 14. The performance parameters of the chickens and tibia bones can be set as indicators or new targets for the clinical diagnosis and prevention of TD in broiler chickens. Tibial dyschondroplasia is a known disorder of fast-growing broiler chickens that affects the long bone, particularly the proximal tibiotarsus. TD is characterized by non-vascularized cartilage, a distended growth plate, tibia bone deformation and lameness [2, 3]. The main clinical features of TD are gait abnormality, reduced feed intake, claudication in one or both legs, ataxia, the inability to stand and walk properly, and ultimately death $[3,17]$. Although the pathogenesis of TD is still not clear, many research directions have been proposed for its pathogenesis and treatment since 1965 . However, recent studies have shown that nutrition, selective breeding and rearing environment affect the incidence of TD [7-9].
However, few studies have systematically examined the change rule of the different indicators of self-healing TD chickens during different periods of development.

Previous studies have shown that a variety of factors change during the incidence of TD. Normal broiler growth plate cartilage has a circular arc with a tidy, smooth edge, and uniform thickness [7]. Shahzad et al. [8] showed that birds in the TD group have developed a lesion filled with cartilaginous material by day 10 ; when the thiram supplement was stopped, the lesion started decreasing, and the growth plate underwent a recovery process and was replaced by bone by day 14 post-hatch [9]. Genin et al. [24] showed that the growth plate width and lameness had no change in normal chickens, while in TD-afflicted chickens, an enlarged growth plate, TD lesions, and lameness occurred.

We checked whether the development of the tibia is correlated with vascular distribution in the tibial growth
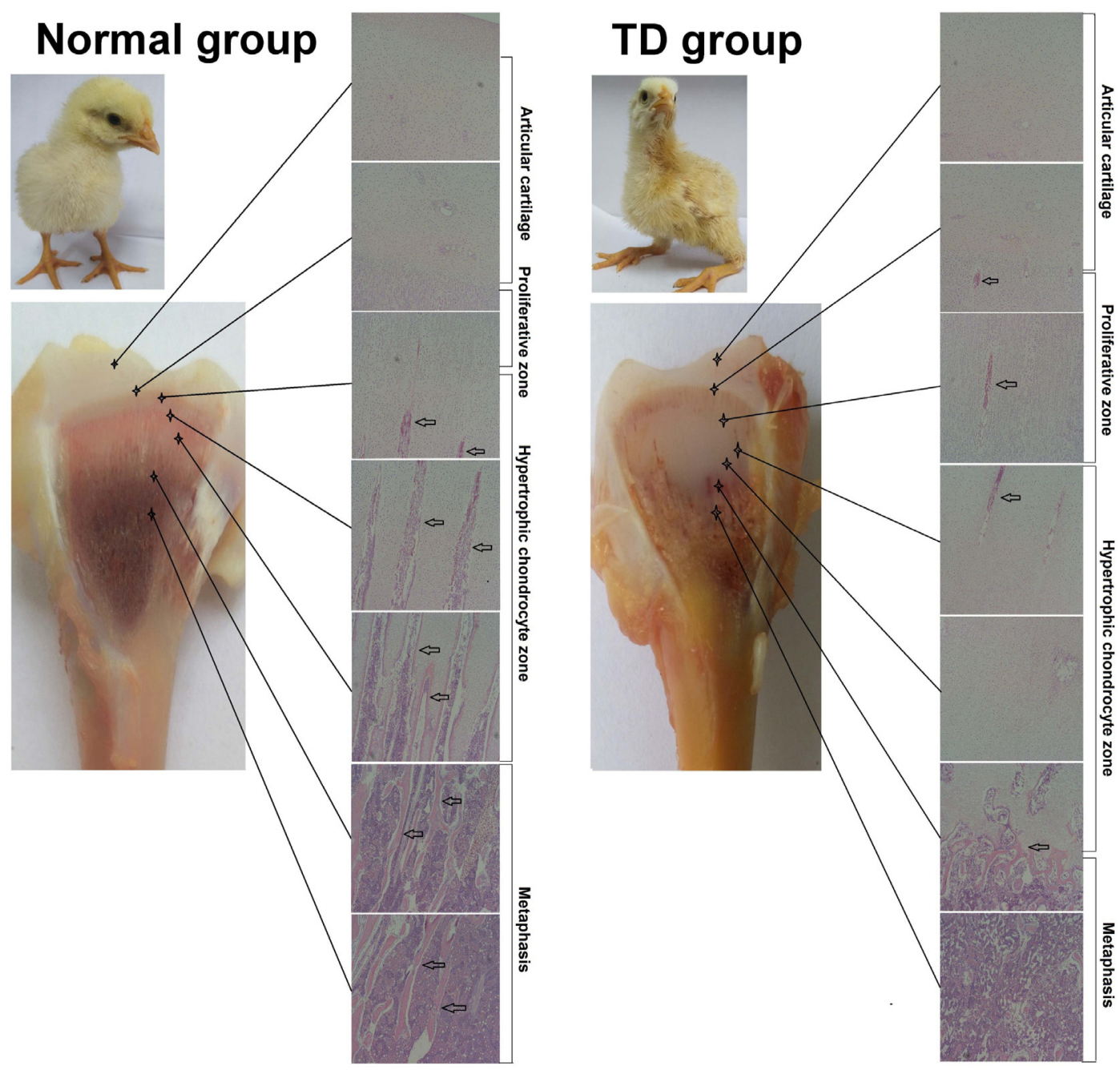

Figure 6: H \& E stained histopathology micrograph depicting the epiphyseal tibial growth plate (GP) in normal and TD chickens. The normal GP exhibits regular columns and cells surrounded by a large number of blood vessels in the proliferative and hypertrophic zones. Necrosis, a reduced number of blood vessels, and non-vascularized GPs were observed in the TD group. Arrow indicates blood vessels. 
Table 1: Serum-biochemical criterion analysis of normal and TD chickens

\begin{tabular}{lccccc}
\hline Parameter & Group & Day 7 & Day 10 & Day 14 & Day 18 \\
\hline ALP (U/L) & Normal group & $132.8 \pm 4.14$ & $141.6 \pm 6.32$ & $153.4 \pm 2.92$ & $172.8 \pm 10.13$ \\
& TD group & $102.6 \pm 4.22^{* *}$ & $105.2 \pm 3.36^{* *}$ & $78.0 \pm 5.25^{* *}$ & $71.6 \pm 4.82^{* *}$ \\
ALT (U/L) & Normal group & $32.9 \pm 2.78$ & $30.6 \pm 5.31$ & $41.7 \pm 6.12$ & $46.2 \pm 3.79$ \\
& TD group & $47.7 \pm 4.27^{*}$ & $68.3 \pm 4.66^{* *}$ & $88.8 \pm 4.35^{* *}$ & $93.0 \pm 1.22^{* *}$ \\
AST (U/L) & Normal group & $51.5 \pm 3.45$ & $56.6 \pm 4.92$ & $61.4 \pm 3.30$ & $67.6 \pm 4.71$ \\
& TD group & $121.0 \pm 6.14^{* *}$ & $137.5 \pm 3.24^{* *}$ & $141.4 \pm 2.42^{* *}$ & $162.5 \pm 7.83^{* *}$ \\
\hline
\end{tabular}

Note: The data are expressed as the mean $\pm \mathrm{SD}$. Student's $t$ test, mean $\pm \mathrm{SD},{ }^{*} p<0.05,{ }^{* *} p<0.01$, normal group vs. TD group.

Table 2: Liver antioxidant activities analysis of normal and TD chickens

\begin{tabular}{lccccc}
\hline Parameter & Group & Day 7 & Day 10 & Day 14 & Day 18 \\
\hline T-AOC (U/mg) & Normal group & $1.47 \pm 0.08$ & $2.24 \pm 0.11$ & $2.57 \pm 0.04$ & $2.34 \pm 0.09$ \\
& TD group & $1.53 \pm 0.03$ & $1.28 \pm 0.06^{* *}$ & $0.80 \pm 0.16^{* *}$ & $1.66 \pm 0.07^{* *}$ \\
SOD (U/mg) & Normal group & $221 \pm 12.7$ & $385 \pm 11.0$ & $309 \pm 6.2$ & $321 \pm 9.8$ \\
& TD group & $173 \pm 11.3^{*}$ & $333 \pm 17.6^{*}$ & $131 \pm 7.3^{* *}$ & $194 \pm 10.1^{* *}$ \\
GSH-Px (U/mg) & Normal group & $17.2 \pm 2.17$ & $37.8 \pm 3.92$ & $41.9 \pm 4.23$ & $52.8 \pm 6.15$ \\
& TD group & $15.7 \pm 1.22$ & $30.5 \pm 4.21^{* *}$ & $31.5 \pm 1.82^{* *}$ & $39.3 \pm 4.09^{* *}$ \\
MDA (nmol/mg) & Normal group & $0.27 \pm 0.022$ & $0.21 \pm 0.026$ & $0.19 \pm 0.015$ & $0.10 \pm 0.011$ \\
& TD group & $0.60 \pm 0.047^{* *}$ & $0.42 \pm 0.038^{* *}$ & $0.38 \pm 0.036^{* *}$ & $0.25 \pm 0.039^{* *}$ \\
\hline
\end{tabular}

Note: The data are expressed as the mean \pm SD. Student's $t$ test, mean $\pm \mathrm{SD}, * P<0.05, * * P<0.01$, normal group vs. TD group.

plate by histological assessments. The results showed that vascular distribution decreased during the study period in the TD group compared with the normal group due to the damaged GP. The cellular morphology in different regions of the growth plate revealed that thiram caused morphological and histological changes characterized by a significantly thickened avascular cartilage plug, dry chondrocytes, and empty cartilage lacunae. Moreover, the particular arrangement and the shape of the cells were compromised due to the lack of blood vessels in the TD affected growth plate [21]. Leach and Nesheim suggested that the TD contributes to the accumulation of cartilage extending from the epiphyseal growth plate into the metaphysis [25]. Thiram causes TD lesions [21] due to mitochondrial injury, membrane damage, inhibition of angiogenesis, and cytotoxic effects [13].

The liver plays an important role in the metabolism and detoxification of biological substances. Reactive oxygen species (ROS), which are constantly produced in animals, serve as a common feature in the "vicious circle" of oxidative stress. The antioxidant enzymes including glutathione peroxidase and superoxide dismutase remove ROS. Thiram has been suggested as a potent oxidative agent; Marikovsky [26] has reported that this compound markedly inhibits SOD activity and rapidly depletes GSH in humans leading to an oxidative imbalance. This type of stress results in the lipid peroxidation of the cell membrane and releases MDA as an end product [3, 27]. In our study, we measured the levels of SOD, GSHPx, T-AOC activity and MDA content to evaluate the antioxidant levels in the liver. Li et al. [23] reported that thiram destroyed the oxidative imbalance by reducing SOD and GSH-Px, inducing oxidative processes in chicken livers such as lipid peroxidation, as evidenced by higher MDA concentrations, affecting liver function and causing TD in broilers in 10 days. Our investigation regarding the changes in antioxidant indicators in the livers of TD chickens indicated that the levels of SOD, T-AOC, GSH-Px and ALP were significantly $(P<0.05)$ decreased compared with those in normal group, while the levels of MDA, AST and ALT were increased significantly $(P<0.05)$ in TD chicks during the study period. The higher serum concentrations of AST and ALT enzymes indicate the release of aminotransferase from hepatocytes into the blood stream due to liver damage. The development of tibial cartilage in birds is coordinated by a variety of enzymes, and most of those enzymes transform, mature and metabolize in the liver. In poultry, the content of ALP can indicate the degree of cartilage cell differentiation and mineralization, and it is closely related to cartilage reconstruction [1, 23]. Furthermore, Rath et al. [21] found that thiram induced TD by a mechanism involving the destruction of blood capillaries and chondrocyte necrosis in tibial growth plates. Consequently, the thiram-fed TD 
chickens had a very high oxidation potential in the liver, and this oxidation could destroy essential cysteine residues in hydroxylases and cause a decrease in enzyme activity. The decrease in hydroxylase in the liver of thiram-fed broilers interferes with the activation of vitamin D and hinders bone calcification and chondrocyte vascularization.

Heat shock protein 90 is a well-conserved stress protein that is highly expressed in various types of stress. Hsp90 plays an important role in bone and muscle development; the level of this protein is highly expressed in cancer tissues and contributes to various disease processes [1, 17]. Hsp90-based therapy is a promising target in clinical practice [28] and is generally highly expressed in the non-vascularized cartilage of TDaffected birds [29]. Therefore, Hsp90 was evaluated to confirm the mRNA transcriptional and translational level in the tibial growth plate of the TD group compared with the normal group. Its expression level was significantly increased in TD chickens during the experimental period, which indicated that Hsp90 plays an important role in stress. Heat-shock protein 90 is a proangiogenic factor in mammalian tissues and in tumors. Hsp90 is implicated in angiogenesis by affecting the VEGF/VEGF-receptor system. Modulation of HIF-1 activity is highly dependent on Hsp90 inhibitor concentrations [1, 29]. Hsp90 and
VEGF are pro-angiogenic regulators [29]. In TD-afflicted birds, the level of Hsp-90 expression determines the vascularization and mineralization degree in chondrocytes rather than the VEGF level [17]. VEGF and its receptors are important for bone growth and development, chondrocyte differentiation and angiogenesis [23]. In the current study, VEFG expression was greatly increased in TD birds compared with that in normal birds. The up-regulation of VEGF during this experiment was concomitant with that observed in previous studies [9, 11, 17, 21, 25, 29]. On other hand Herzog et al. [29] stated that increased blood vessel formation was observed in TD in which the low level of Flk-1 was described as one of the rate-limiting factors of blood-vessel formation that resulted in the unvascularized growth plate. An in vitro model of human chondrocyte culture also showed an increase in the expression level of VEGF [30, 31]. Thiram appeared to be effective in up-regulating VEGF expression, suggesting that thiram has proangiogenic activity [21]. VEGF mRNA transcripts were increased significantly in TD-affected chicks [29]. Dan et al. [6] and Genin et al. [24] found that TD endorsed the change in the VEGF signaling pathway and in abnormal chondrocyte differentiation. Wnt4 is a secreted protein; some research has shown that Wnt4 from osteoblasts significantly

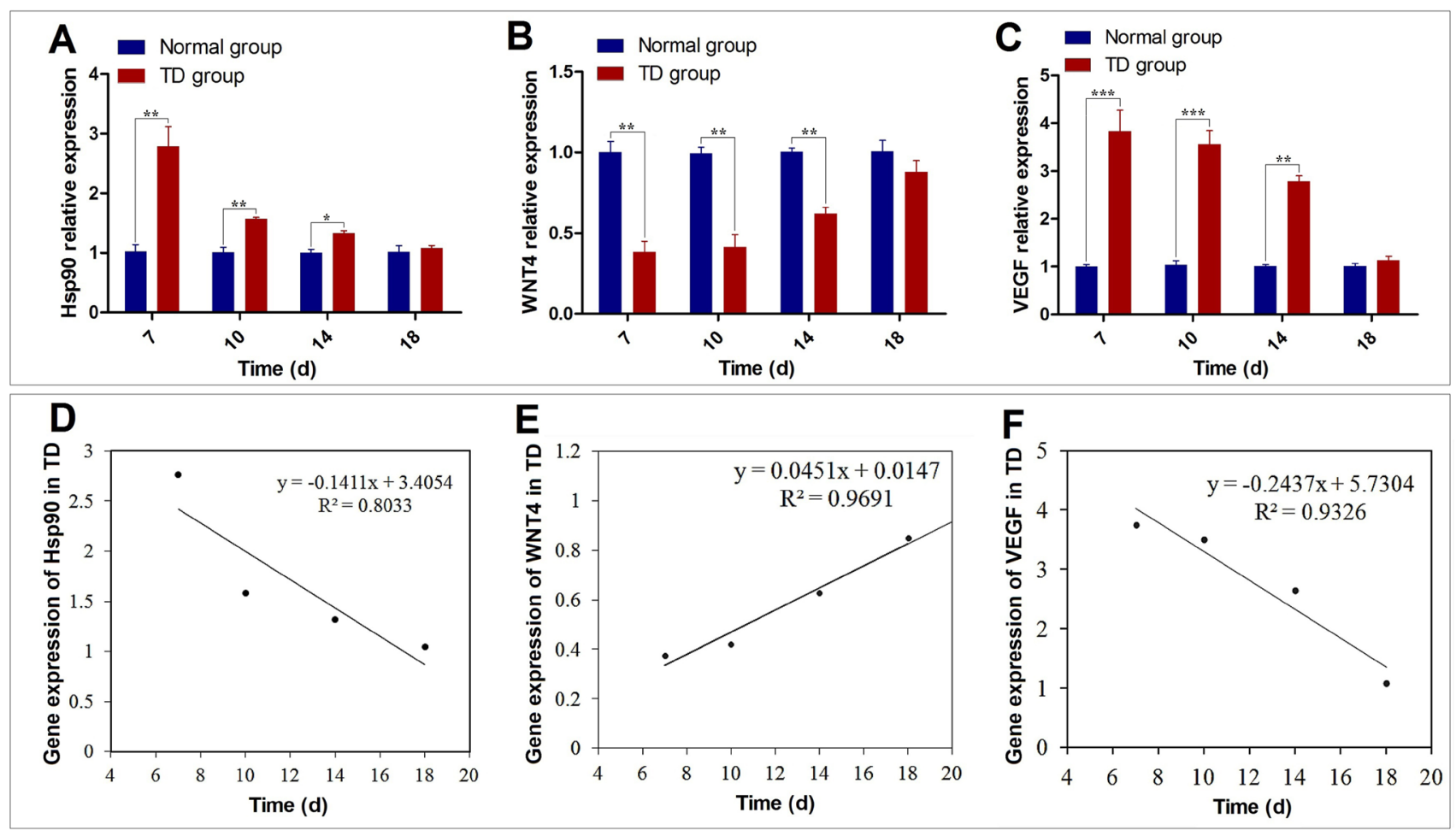

Figure 7: RT-qPCR analysis of Hsp90, WNT4 and VEGF genes in normal and TD chickens. (A-C) The mRNA expressions levels of Hsp90, WNT4 and VEGF genes in the normal and TD group on experimental days 7, 10, 14 and 18 as determined by RT-qPCR. The results are expressed in arbitrary units, and data represent the mean \pm SE. The mRNA expression level is relative to that of the normal group. The normal group expression level values were set to one for comparison as n-fold differences and indicate significant differences $(P<0.05)$. (D-F) Indicates the evaluation of the correlation of Hsp90, WNT4 and VEGF gene expression levels. Student's $t$-test, ${ }^{*} P<0.05$, ${ }^{* *} P<0.01,{ }^{* * *} P<0.001$. Error bars indicate SD. 
protected the tibia from bone loss and inhibited osteoclast formation and bone resorption [19]. In our study, we explored Wnt4 expression in the growth plate of TD chicks. The results showed a significant decrease in Wnt4 levels in TD chickens compared with those in normal chickens from day 7 to day 14, while the expression levels were restored on day 18 . Therefore, the significant decrease in the Wnt4 level served as a genetic indicator of tibia deformation and resorption. It is known that the growth and development of the bone cannot be separated from the nutritional support of the blood [14].

In summary, the results of our systemic study comparing clinical, pathological and genetic indicators in self-healing tibial dyschondroplasia chickens and normal chickens during different age periods reveals a new target for the clinical diagnosis and prevention of TD in broiler chickens.

\section{MATERIALS AND METHODS}

\section{Ethics approval}

All experiments were conducted under the approval and guidelines of the Institutional Animal Welfare and Research Ethics Committee of Huazhong Agricultural University Wuhan, China (approval number: 31272517). All animal experiments and procedures were conducted under the relevant guidelines of the Proclamation of the

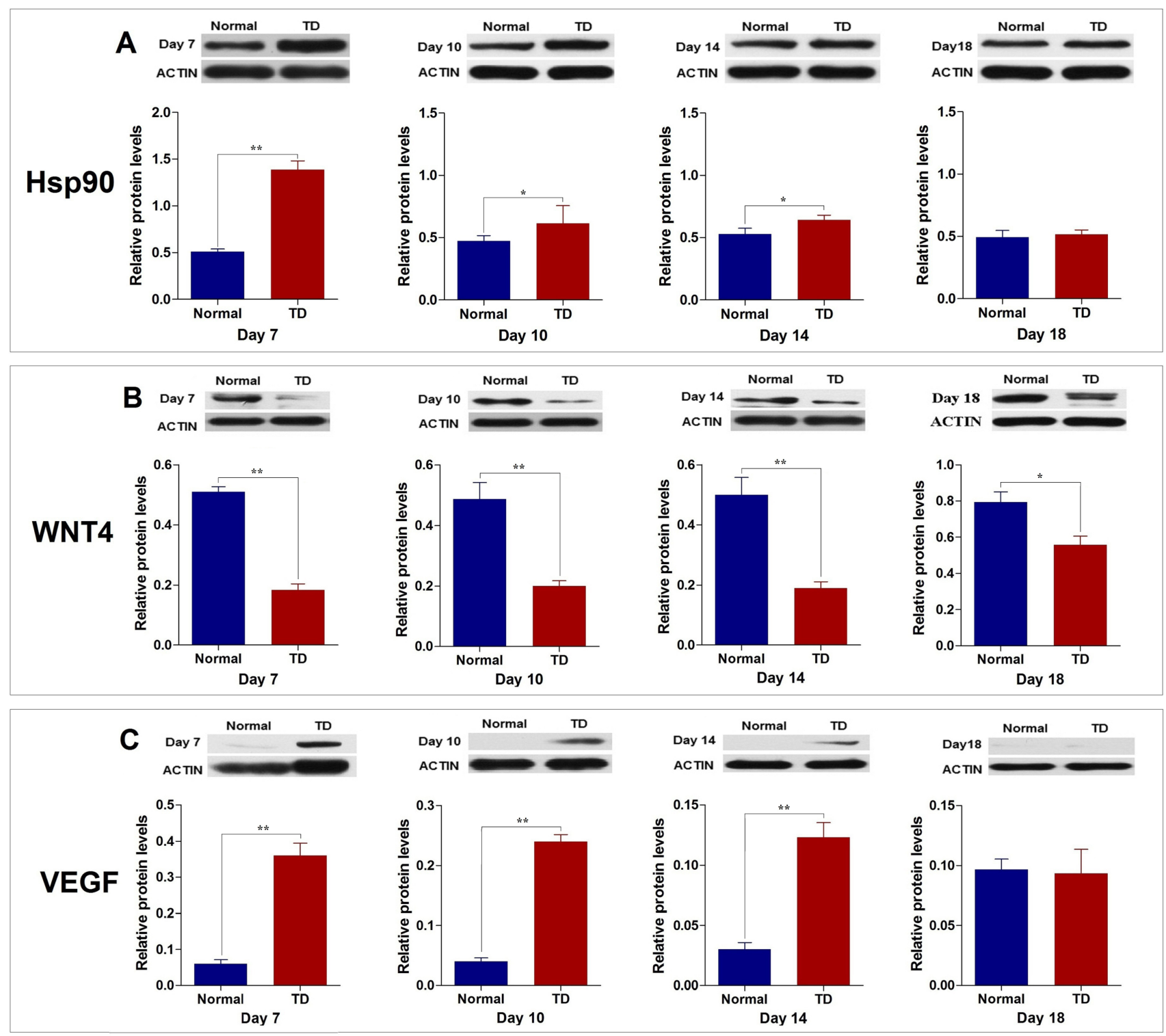

Figure 8: Western blot analysis of Hsp90, WNT4 and VEGF protein expression levels in normal and TD chickens. (A-C) The protein expression levels of Hsp90, WNT4 and VEGF by western blotting in the normal and TD group during experimental days $7,10,14$ and 18 . The results are expressed in arbitrary units, and the data represent the mean \pm SE. The protein expression levels are relative to the normal group. Student's $t$-test, ${ }^{*} P<0.05,{ }^{* *} P<0.01$. 
Standing Committee of Hubei People's Congress (PSCH No. 5), China.

\section{Chicken management and experimental design}

Two hundred 1-day-old Arbor Acres (AA) broiler chickens, weighing $48 \pm 6 \mathrm{~g}$, were purchased from a commercial hatchery (Chia Tai Animal Husbandry Co. Ltd., Wuhan, China). The chickens were maintained and raised under the recommended temperature and standard hygienic conditions (temperature $36^{\circ} \mathrm{C} ; 60 \%$ humidity). After 3 days, the chickens were equally divided into the normal group $(n=100)$ and the TD group $(n=100)$. The normal group was fed a standard normal diet ad libitum as suggested by the National Research Council [32], while the TD group was fed a normal diet supplemented with tetramethylthiuram disulphide (thiram) at $50 \mathrm{mg} / \mathrm{kg}$ of feed. The nutrient composition of the broiler chicken diet is given in Supplementary Table 1. After 7 days, the TD group was fed a standard normal diet without the addition of thiram to observe TD self-healing.

\section{Production parameter analysis and sample collection}

These two groups, the normal group $(n=100)$ and the TD group $(n=100)$, were raised for 18 days, and the number of lame birds (lameness), weight, feed intake and mortality in each group were recorded. The chickens were strictly observed for lameness by visual examination of their movement throughout the experimental period and by TD scoring. During the experimental period, 20 chickens from each group (normal group; TD group) were euthanized randomly on days 7, 10, 14 and 18 . However, before euthanizing, blood was collected from each group of chickens for biochemical analysis. After euthanizing, TD was scored according to Pines et al. [33] and Rath et al. [11]. On days 7, 10,14 and 18, ten tibia bones $(n=10)$ from each group were fixed in $4 \%$ paraformaldehyde (hematoxylin \& eosin staining), and thirty tibia bones $(n=30)$ on days $7,10,14$ and 18 from each group were dissected out; the length, width and weight of the tibia bones along with the width of the tibial growth plate were measured, immediately frozen in liquid nitrogen and stored at $-70^{\circ} \mathrm{C}$ for further analysis (western blotting and RT-qPCR analysis). The liver samples were also dissected, immediately frozen in liquid nitrogen, and then stored at $-70^{\circ} \mathrm{C}$ for later use.

\section{Histology and hematoxylin \& eosin staining}

The tibiotarsal bone $(n=10)$ samples collected on days $7,10,14$ and 18 from each group were fixed overnight in paraformaldehyde $\left(4 \% \mathrm{v} / \mathrm{v}\right.$ in PBS) at $4{ }^{\circ} \mathrm{C}$ and decalcified in ethylenediamine tetraacetic acid $(10 \% \mathrm{v} / \mathrm{v})$. Then, after dehydration in ethanol, clearing in xylene and embedding in paraffin wax, 5 - $\mu$ m-thick histological sections were cut, and the tissue sections were counterstained with hematoxylin \& eosin staining using the method of Tian et al. [7].

\section{Biochemical criterion determination}

The blood samples were centrifuged at $3000 \times \mathrm{g}$ for $20 \mathrm{~min}$ to separate the serum and stored at $-20^{\circ} \mathrm{C}$ until subsequent use and for further analysis of ALT and AST levels and ALP activity. The concentrations of AST, ALP and ALT in the serum samples were measured in both the normal and TD group using a commercial reagent kit (Nanjing Institute of Biological Engineering Inc. Jiangsu, China) via a semi-automatic biochemical machine (Coulter ${ }^{\circledR}$ LH 750, Guangdong). AST, ALT and ALP activities are presented in unit per liter (U/L).

\section{Measurement of SOD, GSH-Px, T-AOC activity and MDA content}

Liver samples were processed to measure the levels of SOD, GSH-Px, T-AOC and MDA activity by using a commercial assay kit (Nanjing Institute of Biological Engineering Inc. Jiangsu, China) in the normal and TD group as mentioned in previous studies [1,23]. Briefly, the livers from each group were rinsed, measured and homogenized for 10 minutes. Then, after centrifugation at $3000 \mathrm{rpm} / \mathrm{min}$ for 10 minutes at $4^{\circ} \mathrm{C}$, SOD, GSH-Px, T-AOC and MDA content levels were assessed. Liver SOD, GSH-Px and T-AOC activities were measured in U per milligram of protein (U/mg protein), and the MDA contents were measured in nanomoles per milligram of protein (nmole/mg protein).

\section{Quantitative reverse transcription-polymerase chain reaction (RT-qPCR)}

Fifteen growth plates $(n=15)$ from each group were frozen in liquid nitrogen on days 7, 10, 14 and 18 and homogenized in TRIzol reagent (Invitrogen, Carlsbad, California, USA) to extract total RNA, which was reverse transcribed to cDNA using a TransScript First-Strand cDNA Synthesis Kit (TransGen Biotech Co. Ltd., Beijing, China) according to the manufacturer's instructions. Reverse transcription was run at reaction temperatures of $42^{\circ} \mathrm{C}$ for $30 \mathrm{~min}$ and $85^{\circ} \mathrm{C}$ for $5 \mathrm{~min}$ in a thermocycler (Applied Biosystems CA, USA), and the synthesis of cDNA was performed in a final reaction volume of $25 \mu 1$.

The RT-qPCR was performed four times with the Step One-Plus ${ }^{\text {TM }}$ Real-Time PCR System (Applied Biosystems, Foster City, CA, USA) for Hsp90, Wnt4, VEGF and glyceraldehyde 3-phosphate dehydrogenase (GAPDH) genes. The primers used for detecting mRNA expression were synthesized by Yingjun (Wuhan, China) and are shown in Table 3. The PCR reactions were run in 
Table 3: Primers used for the Quantitative Polymerase Chain Reaction (qPCR)

\begin{tabular}{|c|c|c|c|c|}
\hline Genes & Accession number & Primer sequence $\left(5^{\prime}-3^{\prime}\right)$ & Product size (bp) & $\begin{array}{c}\text { Tm } \\
\left({ }^{\circ} \mathrm{C}\right)\end{array}$ \\
\hline \multirow{2}{*}{ Hsp90 } & \multirow{2}{*}{ X15028.1 } & F:AGCTCTTAGACTGGCTTGTGT & \multirow{2}{*}{125} & \multirow{2}{*}{59} \\
\hline & & R:TGCTCGGGTCAGTCAAACTC & & \\
\hline \multirow{2}{*}{$V E G F$} & \multirow{2}{*}{ XM_019612783 } & F:AAAGCGAGGAAAGGGGAAGG & \multirow{2}{*}{94} & \multirow{2}{*}{55} \\
\hline & & R:TCTCСТCTCTGAGCAAGGCT & & \\
\hline \multirow{2}{*}{ WNT4 } & \multirow{2}{*}{ NM_204783.1 } & F: AAGGGGCATCTTCCAACAGA & \multirow{2}{*}{136} & \multirow{2}{*}{58} \\
\hline & & R: TTCCAGCACGTCTTGAACTC & & \\
\hline \multirow{2}{*}{$G A P D H$} & \multirow{2}{*}{ XM_019960295 } & F:CCTTCATTGACCTTCACTACATGGTCTA & \multirow{2}{*}{127} & \multirow{2}{*}{58} \\
\hline & & R:TGGAAGATGGTGATGGCCTTTCCATTG & & \\
\hline
\end{tabular}

quadruplicate with the StepOne Plus ${ }^{\mathrm{TM}}$ Real-Time PCR System (Applied Biosystems, Foster City, CA, USA) using SYBR Green RT-PCR Kit (Takara, Dalian China) in a $20 \mu \mathrm{l}$ total reaction volume. The reaction mixture consisted of $2 \mu \mathrm{l}$ of cDNA, specific forward and reverse primers (1 $\mu$ l of each) $10 \mu \mathrm{l}$ of SYBR Premix Ex Taq and the ROX reference dye with the following thermal cycling parameters: $95^{\circ} \mathrm{C}$ for $30 \mathrm{sec}, 40$ amplification cycles at $95^{\circ} \mathrm{C}$ for $8 \mathrm{sec}, 59^{\circ} \mathrm{C}$ for $30 \mathrm{sec}$ and $72^{\circ} \mathrm{C}$ for $30 \mathrm{sec}$. The fluorescence threshold cycle value $(\mathrm{Ct})$ was obtained for each curve and normalized to that obtained for the GAPDH housekeeping gene in the same sample to normalize for discrepancies in sample loading. The differences in $\mathrm{Ct}$ values of the treatment and normal group were then computed and exponentially multiplied to the base of two to obtain the relative differences in expression levels [34].

\section{Western Blot analysis}

Fifteen growth plates from each group $(n=15)$ on days 7, 10, 14 and 18 were homogenized in ice-cold PBS and kept at $4^{\circ} \mathrm{C}$ for $1 \mathrm{~h}$ before they were centrifuged at $17,000 \times \mathrm{g}$ for $20 \mathrm{~min}$ at $4^{\circ} \mathrm{C}$ to collect the supernatant. The protein concentrations of the supernatants were determined by the BCA method (Pierce, Rockford, USA). Protein samples $(40 \mu \mathrm{g})$ were mixed with the loading buffer, electrophoretically separated by SDS-PAGE on $12 \%$ polyacrylamide gels at $100 \mathrm{mV}$ and transferred at $130 \mathrm{mV}$ for $1 \mathrm{~h}$ and $40 \mathrm{~min}$ to nitrocellulose filter membranes (Millipore, BioSharp, Anhui, China). The membranes were incubated in 5\% skimmed milk at room temperature for $1 \mathrm{~h}$ to prevent nonspecific binding and then incubated overnight at $4{ }^{\circ} \mathrm{C}$ with rabbit monoclonal anti-VEGF primary antibodies (1:1000 dilution); rabbit monoclonal anti-Hsp90 primary antibody (1:1000 dilution); or the rabbit monoclonal anti-WNT4 primary antibody (1:1000 dilution) from ABclonal Technology, Wuhan, China. The membranes were washed 3 times with PBS and Tween 20 (0.01 mol/L, $\mathrm{pH} \mathrm{7.4)} \mathrm{for} 5 \mathrm{~min}$ each and then incubated with the secondary rabbit monoclonal anti-VEGF primary antibody (1:1000 dilutions) from ABclonal Technology, Wuhan, China, for 1 hour at room temperature. Finally, the membranes were washed 4 times with TBST again. After washing was complete, the images were captured with an imaging system (UVP, Upland, CA, USA).

\section{Statistical analysis}

Data were examined with two-way ANOVA and by Student's $t$-test to compare the differences between the mean values of the normal and treatment group. The differences were considered statistically significant if $P<0.05$. All statistical analyses were performed using SPSS 19.0 software and the results are presented as the means \pm standard error of the means (S.E.M.).

\section{CONFLICTS OF INTEREST}

None

\section{FUNDING}

This study was supported by National Key Research and Development Program of China (Project No. 2017YFD0502200), National Natural Science Foundation of China (No.31460682), and Research Fund for the Doctoral Program of Higher Education of China (No. 20120146110017).

\section{REFERENCES}

1. Nabi F, Li K, Shahzad M, Han Z, Zhang D, Liu J, Li K. Gambogic acid inhibits hsp90 expressions in thiram-induced tibial dyschondroplasia. Pak Vet J. 2016; 36:224-25.

2. Jefferies D, Houston B, Lester D, Whitehead CC, Thorp $\mathrm{BH}$, Botman M, Farquharson C. Expression patterns of chondrocyte genes cloned by differential display in tibial dyschondroplasia. Biochim Biophys Acta. 2000; 1501:180-88. 
3. Shahzad M, Liu J, Gao J, Wang Z, Zhang D, Nabi F, Li J. Hsp-90 inhibitor geldanamycin attenuates liver oxidative stress and toxicity in thiram-induced tibial dyschondroplasia. Pak Vet J. 2014; 34:545-47.

4. Edwards HM Jr. Nutrition and skeletal problems in poultry. Poult Sci. 2000; 79:1018-23.

5. Li J, Bi D, Pan S, Zhang Y, Zhou D. Effects of high dietary vitamin A supplementation on tibial dyschondroplasia, skin pigmentation and growth performance in avian broilers. Res Vet Sci. 2008; 84:409-12.

6. Dan H, Simsa-Maziel S, Hisdai A, Sela-Donenfeld D, Monsonego Ornan E. Expression of matrix metalloproteinases during impairment and recovery of the avian growth plate. J Anim Sci. 2009; 87:3544-55.

7. Tian WX, Li JK, Qin P, Wang R, Ning GB, Qiao JG, Li HQ, Bi DR, Pan SY, Guo DZ. Screening of differentially expressed genes in the growth plate of broiler chickens with tibial dyschondroplasia by microarray analysis. BMC Genomics. 2013; 14:276.

8. Shahzad M, Gao J, Qin P, Liu J, Wang Z, Zhang D, Li J. Expression of genes encoding matrilin-3 and cyclin-I during the impairment and recovery of chicken growth plate in tibial dyschondroplasia. Avian Dis. 2014; 58:468-73.

9. Shahzad M, Liu J, Gao J, Wang Z, Zhang D, Nabi F, Li K, Li J. Differential expression of extracellular matrix metalloproteinase inducer (EMMPRIN/CD147) in avian tibial dyschondroplasia. Avian Pathol. 2015; 44:13-18.

10. Börjesson AE, Lagerquist MK, Windahl SH, Ohlsson C. The role of estrogen receptor $\alpha$ in the regulation of bone and growth plate cartilage. Cell Mol Life Sci. 2013; 70:4023-37.

11. Rath NC, Huff WE, Huff GR. Thiram-induced changes in the expression of genes relating to vascularization and tibial dyschondroplasia. Poult Sci. 2007; 86:2390-95.

12. Velada I, Capela-Silva F, Reis F, Pires E, Egas C, RodriguesSantos P, Barros MT. Expression of genes encoding extracellular matrix macromolecules and metalloproteinases in avian tibial dyschondroplasia. J Comp Pathol. 2011; 145:174-86.

13. Shim MY, Karnuah AB, Anthony NB, Pesti GM, Aggrey SE. The effects of broiler chicken growth rate on valgus, varus, and tibial dyschondroplasia. Poult Sci. 2012; 91:62-65.

14. Filipowska J, Tomaszewski KA. NiedZwiedzki L, Walocha JA, Niedzwiedzki T. The role of vasculature in bone development, regeneration and proper systemic 400 functioning. Angiogenesis. 2017; 20:291-302.

15. Mehmood K, Zhang H, Iqbal MK, Rehman MU, Shahzad M, Li K, Huang S, Nabi F, Zhang L, Li J. In Vitro effect of apigenin and danshen in tibial dyschondroplasia through inhibition of heat-shock protein 90 and vascular endothelial growth factor expressions in avian growth plate cells. Avian Dis. 2017; 61:372-377.
16. Praul CA, Ford BC, Gay CV, Pines M, Leach RM. Gene expression and tibial dyschondroplasia. Poult Sci. 2000; 79:1009-13.

17. Nabi F, Shahzad M, Liu J, Li K, Han Z, Zhang D, Iqbal MK, Li J. Hsp90 inhibitor celastrol reinstates growth plate angiogenesis in thiram-induced tibial dyschondroplasia. Avian Pathol. 2016; 45:187-93.

18. Chung UI, Lanske B, Lee K, Li E, Kronenberg H. The parathyroid hormone/parathyroid hormone-related peptide receptor coordinates endochondral bone development by directly controlling chondrocyte differentiation. Proc Natl Acad Sci USA. 1998; 95:13030-35.

19. Hartmann C, Tabin CJ. Dual roles of Wnt signaling during chondrogenesis in the chicken limb. Development. 2000; 127:3141-59.

20. Zhang JP, Deng YF, Zhou ZL, Hou JF. Expression and identification of recombinant chicken vascular endothelial growth factor in Pichia pastoris and its role in the pathogenesis of tibial dyschondroplasia. Poult Sci. 2013; 92:3214-27.

21. Rath NC, Richards MP, Huff WE, Huff GR, Balog JM. Changes in the tibial growth plates of chickens with thiram-induced dyschondroplasia. J Comp Pathol. 2005; 133:41-52.

22. Chen H, Ghori-Javed FY, Rashid H, Adhami MD, Serra $\mathrm{R}$, Gutierrez SE, Javed A. Runx2 regulates endochondral ossification through control of chondrocyte proliferation and differentiation. J Bone Miner Res. 2014; 29:2653-65.

23. Li J, Bi D, Pan S, Zhang Y. Effect of diet with thiram on liver antioxidant capacity and tibial dyschondroplasia in broilers. Br Poult Sci. 2007; 48:724-28.

24. Genin O, Hasdai A, Shinder D, Pines M. The effect of inhibition of heat-shock proteins on thiram-induced tibial dyschondroplasia. Poult Sci. 2012; 91:1619-26.

25. Leach RM Jr, Nesheim MC. Nutritional genetic and morphological studies of an abnormal cartilage formation in young chicken. J Nutr. 1965; 86:236-44.

26. Marikovsky M. Thiram inhibits angiogenesis and slows the development of experimental tumours in mice. Br J Cancer. 2002; 86:779-87.

27. Perry JJ, Shin DS, Getzoff ED, Tainer JA. The structural biochemistry of the superoxide dismutases. Biochim Biophys Acta. 2010; 1804:245-62.

28. Csermely P, Schnaider T, Soti C, Prohászka Z, Nardai G. The $90-\mathrm{kDa}$ molecular chaperone family: structure, function, and clinical applications. A comprehensive review. Pharmacol Ther. 1998; 79:129-68.

29. Herzog A, Genin O, Hasdai A, Shinder D, Pines M. Hsp90 and angiogenesis in bone disorders-lessons from the avian growth plate. Am J Physiol Regul Integr Comp Physiol. 2011; 301:R140-47.

30. Beckmann R, Houben A, Tohidnezhad M, Kweider N, Fragoulis A, Wruck CJ, Brandenburg LO, HermannsSachweh B, Goldring MB, Pufe T, Jahr H. Mechanical 
forces induce changes in VEGF and VEGFR-1/sFlt-1 expression in human chondrocytes. Int J Mol Sci. 2014; 15:15456-74.

31. Schnabel M, Marlovits S, Eckhoff G, Fichtel I, Gotzen L, Vécsei V, Schlegel J. Dedifferentiation-associated changes in morphology and gene expression in primary human articular chondrocytes in cell culture. Osteoarthritis Cartilage. 2002; 10:62-70.

32. NRC (National Research Council). Nutrient requirements of poultry. 9th Rev. Ed. Washington (DC): National Academy Press. 2001.
33. Pines M, Hasdai A, Monsonego-Ornan M. Tibial dyschondroplasia-tools, new insights and future prospects. Worlds Poult Sci J. 2005; 61:285-97.

34. Livak KJ, Schmittgen TD. Analysis of relative gene expression data using real-time quantitative PCR and the 2(-Delta Delta C(T)) Method. Methods. 2001; 25:402-08. 